Kansas State University Libraries

New Prairie Press

\title{
EXPERIMENTAL ERROR IN AGRONOMIC FIELD TRIALS
}

Thomas M. Loughin

D. F. Cox

Paul N. Hinz

William T. Schapaugh Jr.

Lora Kilgore-Norquest

See next page for additional authors

Follow this and additional works at: https://newprairiepress.org/agstatconference

Part of the Agriculture Commons, and the Applied Statistics Commons

\section{(c) (1) ()}

This work is licensed under a Creative Commons Attribution-Noncommercial-No Derivative Works 4.0 License.

\section{Recommended Citation}

Loughin, Thomas M.; Cox, D. F.; Hinz, Paul N.; Schapaugh, William T. Jr.; and Kilgore-Norquest, Lora (1994). "EXPERIMENTAL ERROR IN AGRONOMIC FIELD TRIALS," Conference on Applied Statistics in Agriculture. https://doi.org/10.4148/2475-7772.1356

This is brought to you for free and open access by the Conferences at New Prairie Press. It has been accepted for inclusion in Conference on Applied Statistics in Agriculture by an authorized administrator of New Prairie Press. For more information, please contact cads@k-state.edu. 
Author Information

Thomas M. Loughin, D. F. Cox, Paul N. Hinz, William T. Schapaugh Jr., and Lora Kilgore-Norquest 


\title{
EXPERIMENTAL ERROR IN AGRONOMIC FIELD TRIALS
}

\author{
Thomas M. Loughin \\ Statistical Laboratory and Department of Statistics \\ Kansas State University, Manhattan, KS 66506 \\ D. F. Cox, Paul N. Hinz \\ Statistical Laboratory and Department of Statistics \\ Iowa State University, Ames, IA 50011 \\ William T. Schapaugh, Jr., Lora Kilgore-Norquest \\ Agronomy Department \\ Kansas State University, Manhattan, KS 66506
}

\begin{abstract}
Agronomic experiments often summarize work carried out in trials run in several locations over several years, referred to generically as environments. The appropriate statistical analyses for these experiments depend on definitions used for experimental error. The results of one such experiment, in which identical designs were used in each environment, illustrate the commonalities and differences in analyses that can result from using different definitions of experimental error.
\end{abstract}

\section{INTRODUCTION}

Performing useful statistical analyses often requires a compromise. In order to clearly describe the trends contained within a set of data, an analysis must be both accurate and precise. To attain higher levels of accuracy and precision leads to greater complexity in the statistical analysis. Unfortunately, the more technically complete analyses sometimes provide results that are not easily interpreted. On the other hand, oversimplification can render an analysis ineffective by disguising real and important trends in the data.

Agronomic field trials often result in analyses of at least moderate complexity. Trials are often replicated in several locations (usually representing a region in which the treatments could be considered for use) and over several years. Typically, the same set of treatments in the same experimental design is used in each year at each location. These year-by-location combinations are generically referred to as "environments."

The data from these experiments are combined into a single analysis to assess overall treatment effects and how much these effects vary across environments. As a result there are usually at least two sources of experimental variability which must 
be used in the analysis. These correspond to the different levels of replication under which the trials were carried out and can be classified as "within-environment" sources and "across-environment" sources. The manner in which these various sources are used in definitions of experimental error depends greatly upon the degree to which simplicity is favored over completeness.

In the past, the lack of readily available computational methods for the more complicated analyses made the statistical simplification of complex experiments an important step. Even for a simplified analysis, however, some standard statistical packages may base their computations on assumptions about the inference space (McLean, Sanders, and Stroup, 1991) or the estimability of contrasts (Milliken, 1992) that may not be appropriate. Now, with the increasing availability of mixed model software, such as PROC MIXED (SAS Institute, 1992), there are more options for potential analysis methods for designed experiments.

Even so, care must be taken to accurately reflect the nature of the effects which are used in the analysis. The distinction between considering an effect fixed or random is not always very clear, but as McLean, et al. (1991) suggest, the decision for a given effect can have a tremendous impact on the resulting analysis and its interpretation.

In this paper, the effects of the three choices outlined above - complexity of the model, identification of fixed and random effects, and selection of statistical software - are investigated as they relate to definitions of experimental error in a particular agronomic field trial.

\section{EXAMPLE: DETERMINATE/INDETERMI- NATE SOYBEAN TRIALS}

The example studied in this paper is a trial designed to evaluate the effects of of two genetically-controlled growth habits, determinate and indeterminate stem termination, on yield of soybean. Additionally, since 1991 was a drought year in Kansas, there was interest in comparing the impact of two growth habits on yield response under drought stress.

The trial was performed over three years (1990, 1991, and 1992) at three experimental fields in Kansas (Ashland, Hesston, and Ottawa) in such a way that five combinations of year and location were available (Ashland was used only in 1990 and was the only location in use at that time). Twenty near-isogenic lines (or "entries") of soybean were selected for the study and bred so that both stem terminations were present in each entry. In each environment, the experiment had a split-plot design with each of the 20 entries planted in a whole plot consisting of four rows. Each plot was split in half across these rows and the two growth habits were randomly assigned 
to halves of the plot. The center two rows were harvested for seed yield. There were three full blocks of this design in each environment.

\section{METHODS OF ANALYSIS}

The data from this experiment are analyzed in a variety of ways to emphasize the impact that the choice of analysis method can have on the results. The labels HABIT, ENV, ENTRY, and BLOCK (ENV) correspond to growth habits, environments, entries and blocks within environments, respectively.

\subsection{Random and Fixed Effects}

The distinction between fixed and random effects often requires careful thought. In the present study, few would argue against taking BLOCK (ENV) as random and HABIT as fixed. Also, the reasoning behind using the 20 lines of soybean chosen for this experiment had more to do with their representation of genotypic variability than with any distinctive features of the lines themselves, so that ENTRY is best treated as a random effect.

The designation of the effect of the environment classification is less clear, although it is crucial in defining the inference space for which the results are intended. One possibility is that the three locations might be intended to represent some population of possible locations in which the study treatments could be applied. In that case the inference space is broad, since inferences are to be extended to all locations in the population. Because no control can be affected over circumstances from year to year, ENV would certainly be considered a random effect.

Another possibility is that each location might have been chosen to represent conditions (e.g., rainfall, soil, or crop rotation) peculiar to that region. In addition, 1991 was a drought year, and some interest lay in examining the relative effects of HABIT under such stressful conditions. These circumstances would lead to treating ENV as a fixed effect. Inferences are then to be applied only to those particular conditions represented by the environments in the study, and so the inference space is narrow. For the purposes of this exposition, both interpretations for the environment effect are considered and compared.

A third possible interpretation could have considered treating environments as a random effect while wishing to compare the habit effects in drought vs. non-drought conditions. This requires writing contrasts on levels of the HABIT*ENV interaction, despite the fact that this is customarily assumed to be random if ENV is random. Treating as fixed an interaction of a random main effect is an example of an intermediate inference space as defined by McLean, Sanders, and Stroup (1991). 


\subsection{Selection of Models}

A variety of analysis of variance models could be reasonably proposed for this study, with varying complexities and goals. The most direct of these is perhaps the "full model" (FM) approach, which combines the five identical split-plot designs into one analysis. One possible model for this is given, for example, by McIntosh (1983). A slightly different FM approach is applied here in that the HABIT*BLOCK (ENV) is isolated from the subplot error term (see Table 2).

The designation of ENV as a fixed or random effect has a profound effect on the analysis through the FM model. With ENV random, much of the experimental error is derived from the variability of the other effects across environments. When it is fixed, however, the variability of effects across blocks within environments provides the primary measure of error.

One common simplification of the FM model is to eliminate the effect of withinenvironment variability and consider the across-environment variability as the experimental error. This is achieved by reducing the data from the different blocks to a single set of values for each environment, the entry-habit means, for each environment. This is referred to here as the "means over blocks" (MOB) approach. Although this assumes that the environments are indeed random effects, the results of this simplification with fixed ENV will also be noted.

The third approach to be considered is perhaps the most obvious: analyze the split-plot designs separately within each environment. This "by-environment" (BE) approach might arise naturally as a follow-up to either of the other two models, but technically this use is appropriate primarily when environments are considered fixed. The across-environment variability is completely ignored in this approach, so that experimental error is defined through the blocks within each environment. Note that in the FM approach the variability of blocks is assumed to be the same within each environment. In the BE approach, however, error due to blocks may differ among environments.

While numerous other models could be (and in fact were) considered, only the results of the $\mathrm{FM}, \mathrm{MOB}$, and $\mathrm{BE}$ approaches are compared here.

\subsection{Computational Methods}

Combining the two ENV classifications as in Section 3.1 with the possible models described in Section 3.2 yields five analysis settings to be consider. Each of these is analyzed using the two different SAS procedures, PROC GLM and PROC MIXED. These procedures differ in numerous ways, as detailed in the extensive documentation for PROC MIXED (SAS Institute, 1992). The important differences between them as regards the present work are briefly outlined in Table 1. 
F-tests corresponding to both fixed and random effects are automatically provided for each effect using PROC GLM with the TEST option. Denominator degrees of freedom are approximated using Satterthwaite's method (Milliken and Johnson, 1992). The PROC MIXED analyses also use F-tests, but only for all fixed effects in the model, and the F-statistics are not necessarily the same as in PROC GLM . The denominator degrees of freedom are found using the following "containment method" (SAS Institute, 1992): for each fixed effect, if there is a single random effect which can serve as an error term, then the denominator degrees of freedom are those from this random effect. Otherwise, PROC MIXED uses $n-r-1$, where $n$ is the sample size and $r$ is the number of model degrees of freedom allotted to fixed effects. Wald tests based on asymptotic normal theory are automatically provided for all random effects. Unfortunately, these are notoriously unreliable in small samples (SAS Institute, 1992). As an alternative, it is possible to obtain likelihood ratio tests by repeatedly running PROC MIXED on the same model, but with selected random effects omitted. For large models this can result in considerable extra computation.

\section{RESULTS}

The results of the ten analyses are given below. They are first discussed separately for each modeling approach. An $\alpha=.05$ level of significance is used for assessing the importance of all effects.

\subsection{Full Model}

Variance component estimates using the default methods (Table 1) from PROC GLM and PROC MIXED are given in Table 2. For all random effects the two methods produce identical estimates in this balanced data set.

When ENV is considered random, it has by orders of magnitude the largest variance component. Two of the three tests of significance (Table 3) indicate the importance of the effects of environments on yield; the Wald test for this effect fails to find it significant $(p=.16)$. Considering the means in Table 10, it is difficult to accept that the environments - in particular the two drought-year environments - imparted insignificant variability into the soybean yields. In view of the Wald test's documented poor performance in problems of this kind, no further comments will be offered on results from this testing method, despite the fact that it is the default option in PROC MIXED.

The three-way interaction, HABIT*ENTRY*ENV, is strongly significant $(p<.01)$, indicating that the relationships among the environments, among the entries, and between the two growth habits may be somewhat complex. In practice, investigation 
of this interaction, for example through profile plots or contrasts, should precede interpretation of the significance tests for main effects and smaller-order interactions. Briefly, the significance of this three-factor interaction implies that the pattern of growth habit differences in the five environments (see Table 10) is not the same for all entries. A more detailed analysis of these differences is omitted from the present discussion.

In the random environment case, tests for the remaining effects are the same or quite similar for both the PROC GLM and the likelihood ratio analysis from PROC MIXED. The lone fixed effect, HABIT, is nonsignificant, although in light of the significant interaction noted above this does not imply that growth habits have no impact on soybean yields. When ENV is considered fixed, two important changes occur in the significance tests. First, the HABIT effect is now found to be significant by both testing procedures due to the changing structure of variance components which go into making up its error term, the most important of which is the substitution of HABIT*BLOCK (ENV) $(\mathrm{MS}=7)$ for HABIT*ENV $(\mathrm{MS}=102)$. Second, the PROC GLM tests for HABIT and ENV main effects are dependent upon the assumption that there is no $H A B I T * E N V$ interaction. Since the corresponding test of this interaction strongly suggests that this assumption is not satisfied, the interpretations of these main-effect tests are unclear. However, proper investigation of the HABIT*ENV interaction, as in Table 10, may supersede a need for consideration of these two tests.

\subsection{Means over Blocks}

Estimated variance components and $p$-values for tests from the MOB approach are given in Tables 4 and 5, respectively. Variance components from both estimation procedures are again quite similar to each other and change little from those obtained in the FM approach. An important difference between the FM and MOB approaches is that HABIT*ENTRY*ENV serves as a residual error term in the latter, and so no reliable test of significance can be performed for it. Otherwise, test results from this model are essentially the same as those from the previous model. When ENV is random, PROC GLM tests for ENTRY and HABIT are identical to those from the FM approach, since both models use the same error terms. The fixed-environment test for HABIT does use a simpler error term in the MOB analysis, but for these data there is little change in the result.

Another form of MOB model that is sometimes used treats the means as having originated from a split-plot design with environments as a blocking variable, entries as a whole-plot effects, and growth habits as a subplot effect. This differs from the present MOB approach only in that the HABIT*ENV effect joins the three-way interaction in the residual error. Since a separate assessment was desired of the possibly differing growth habit effects in the different environments, this split-plot 
MOB model is not of particular interest to this study. Nonetheless its application reveals an interesting departure from the original MOB approach in that it finds HABIT to be a significant effect even when environments are random. Thus it is evident that even seemingly minor differences in the identification of experimental errors may play a large role in the conclusions that are ultimately drawn.

\subsection{By Environment}

Table 6 provides REML variance component estimates for each of the five split-plot experiments in the study. The environment labels correspond to the three locations (ASHland, HESston, and OTTawa) and to the three years (1990, 1991, and 1992) in which the study was run. Method of moments estimates are identical in all but the footnoted cases, where they differed by no more than 0.2 from their REML counterparts. Differences between estimates from the two methods stem from the fact that MOM sometimesprovides an actual negative value as the estimate for a variance component while REML sets the corresponding estimate to zero.

Results of tests based on these effects are presented in Tables 7-9. As before the F-tests from PROC GLM and the likelihood ratio tests from PROC MIXED are largely in agreement. In each environment there appears to be a significant (or near-significant) variance component for HABIT*ENTRY, indicating that growth habit effects are not the same for all entries. Despite this variability, a significant HABIT effect is still found in two of the five environments. Both the PROC GLM F-tests and the likelihood ratio tests find significant ENTRY in only one environment.

\section{DISCUSSION}

The primary goal in this experiment - the comparison of the two growth habits across the different environments and entries - can be carried out effectively using any of the three approaches from Section 3. Each approach has its strengths and weaknesses.

Among the approaches studied here, the FM approach uses the most detailed definitions of experimental error, providing the most complete foundation for investigating the various growth habit interactions. However, it is also the most difficult to work with for breaking apart and examining these interactions. This is due in part to the complexity of the definition of experimental error for these interactions which follows from this approach. PROC MIXED has been designed to make this kind of work easier by providing less restrictive estimability requirements and more accurate standard errors for least-squares means and contrasts than are available in PROC GLM (Milliken, 1992). However, PROC MIXED can be rather cumbersome to work with if 
tests of random effects are desired, and it will not provide means and standard errors for random effects.

The MOB approach simplifies the analysis of some of the HABIT interactions at the cost of being unable to test the three-way interaction. This simplification is particularly useful in the fixed-environment setting, where the experimental error terms for HABIT, HABIT*ENTRY, and HABIT*ENV are all single mean squares, rather than the linear combinations of mean squares as in the FM approach.

In this example, both the MOB and the FM approaches indicate a need for examining the changes of growth habit effects across environments. Thus, in both cases the $\mathrm{BE}$ approach is a reasonable next step.

Table 10 represents one summary from the BE approach, and it provides interesting and potentially useful information about the growth habits' tendencies in different environments. Specifically, this table points out that the largest differences between the two growth habits were observed in the two drought-year environments (1991). In particular, the indeterminate stem termination growth habit provides, on average over the different lines of soybeans, higher yields than the determinate stem termination in these environments. A more detailed analysis of this interaction might include contrasts comparing the habit effects in the two drought-year environments with those in the other three, using either the FM or MOB approach.

Also, although the MOB approach provides no test for the three-way interaction, the $B E$ results suggest the possibility of such an effect with the detection of HABIT*ENTRY interactions in each environment. Further investigation of these interactions might be done in each environment using profile plots or by examining the differences in yield between the two growth habits over the 20 entries. Results of these detailed analyses are not presented here.

As detailed in Section 3.1, the drawback to applying the BE approach is that, since all experimental errors are based on within-environment variability, inferences are made in the "narrow inference space" as defined by McLean, Sanders, and Stroup (1991). Thus conclusions drawn within an environment apply only to that environment. Patterns may be noted across the environments, but they are not explicitly tested using an across-environment experimental error unless the BE analyses are used in conjunction with another approach.

\section{REFERENCES}

McIntosh M. S. (1983). Analysis of Combined Experiments. Agronomy Journal, 75, $153-155$.

McLean, R. A., Sanders, W. L., and Stroup, W. W. (1991). A Unified Approach to 
Mixed Linear Models. American Statistician, 45, 54-63.

Milliken, G. A. (1992) Analysis of Mixed Models without Mixed Model Software. Proceedings of the $4^{\text {th }}$ Conference on Applies Statistics in Agriculture, 180-189.

Milliken, G. A. and Johnson, D. E. (1992). Analysis of Messy Data, Volume 1: Designed Experiments. New York: Chapman and Hall.

SAS Institute (1992). SAS Technical Report P-229, SAS/STAT Software: Changes and Enhancements, Release 6.0\%. Cary, NC: SAS Institute.

Wolfinger, R., Miles-McDermott, N., and Kendall, J. (1992). Analyzing Split-Plot and Repeated Measures Designs Using Mixed Models. Proceedings of the $4^{\text {th }}$ Conference on Applies Statistics in Agriculture, 190-200. 
Table 1: Comparison of PROC GLM and PROC MIXED

- PROC GLM with RANDOM statement and TEST option

- "Usual" estimation of ANOVA mean squares through least squares

- Automatic computation of table of Expected Mean Squares

- Estimation of variance components for random effects by Method of Moments

- Fixed and random effects tested using F-test

* Use approximated DF

* Appropriate linear combinations of variance components form error terms

- PROC MIXED

- Estimation of variance components for random effects by REML (Wolfinger, et al, 1992)

- Obtain estimate of full covariance matrix for model

- Estimation of fixed effects using weighted least squares

- Tests for effects

* Random-Asymptotic likelihood theory (Normality)

* Fixed-F-test with approximate DF from appropriate error term 
Table 2: Mean squares and variance components for FM approach

\begin{tabular}{|c|c|c|c|c|c|}
\hline \multirow[b]{3}{*}{ Source } & \multicolumn{3}{|c|}{ GLM } & \multicolumn{2}{|c|}{ MIXED } \\
\hline & & Mean & Variance & Variance & Standard \\
\hline & $\mathrm{DF}$ & Square & Component & Component & Error \\
\hline $\mathrm{ENV}^{a}$ & 4 & 20596 & 169.5 & 169.5 & 121.4 \\
\hline BLOCK(ENV) & 10 & 140 & 3.2 & 3.2 & 1.6 \\
\hline ENTRY & 19 & 89 & 0.54 & 0.54 & 1.1 \\
\hline ENTRY*ENV & 76 & 35 & 2.8 & 2.8 & 1.0 \\
\hline $\mathrm{ENTRY}^{*} \mathrm{BLOCK}(\mathrm{ENV})$ & 190 & 10 & 2.1 & 2.1 & 0.6 \\
\hline $\mathrm{HABIT}^{b}$ & 1 & 245 & - & - & - \\
\hline $\mathrm{HABIT}^{*} \mathrm{ENV}^{a}$ & 4 & 102 & 1.5 & 1.5 & 1.2 \\
\hline $\mathrm{HABIT}^{*} \mathrm{BLOCK}(\mathrm{ENV})$ & 10 & 7 & 0.044 & 0.044 & 0.2 \\
\hline HABIT*ENTRY & 19 & 51 & 2.5 & 2.5 & 1.1 \\
\hline $\mathrm{HABIT}^{*} \mathrm{ENTRY}^{*} \mathrm{ENV}$ & 76 & 14 & 2.5 & 2.5 & 0.8 \\
\hline RESIDUAL/ERROR & 190 & 6 & 6.3 & 6.3 & 0.6 \\
\hline
\end{tabular}

${ }^{a}$ Variance component estimated only when considered random

${ }^{b}$ Fixed effect-no variance component estimated

Table 3: P-values for tests of effects from FM approach

\begin{tabular}{|c|c|c|c|c|c|}
\hline \multirow[b]{3}{*}{ Source } & \multicolumn{3}{|c|}{ ENV Random } & \multicolumn{2}{|c|}{ ENV Fixed $^{a}$} \\
\hline & \multirow[b]{2}{*}{ GLM } & \multicolumn{2}{|c|}{ MIXED } & \multirow[b]{2}{*}{ GLM } & \multirow[b]{2}{*}{ MIXED } \\
\hline & & \multicolumn{2}{|l|}{ Likelihood } & & \\
\hline ENV & $<.01$ & $<.01$ & .16 & $<.01^{b}$ & $<.01$ \\
\hline BLOCK(ENV) & $<.01$ & $<.01$ & .04 & & \\
\hline ENTRY & .30 & .62 & .63 & & \\
\hline ENTRY*ENV & $<.01$ & $<.01$ & .01 & & \\
\hline ENTRY*BLOCK(ENV) & $<.01$ & $<.01$ & $<.01$ & & \\
\hline HABIT & .23 & \multicolumn{2}{|c|}{$.19^{c}$} & $.04^{b}$ & .03 \\
\hline $\mathrm{HABIT}^{*} \mathrm{ENV}^{a}$ & $<.01$ & $<.01$ & .23 & $<.01$ & $<.01$ \\
\hline $\mathrm{HABIT}^{*} \mathrm{BLOCK}(\mathrm{ENV})$ & .33 & .77 & .79 & & \\
\hline HABIT*ENTRY & $<.01$ & $<.01$ & .02 & & \\
\hline $\mathrm{HABIT}^{*} \mathrm{ENTRY}{ }^{*} \mathrm{ENV}$ & $<.01$ & $<.01$ & $<.01$ & & \\
\hline
\end{tabular}

\footnotetext{
${ }^{a}$ P-values are presented only for effects for which test statistics change

${ }^{b}$ Requires that ENV*HABIT effect is zero

${ }^{c}$ PROC MIXED test for fixed effects does not depend on method chosen for testing random effects
} 
Table 4: Mean squares and variance components for MOB approach

\begin{tabular}{|c|c|c|c|c|c|}
\hline \multirow[b]{3}{*}{ Source } & \multicolumn{3}{|c|}{ GLM } & \multicolumn{2}{|c|}{ MIXED } \\
\hline & & Mean & Variance & Variance & Standard \\
\hline & DF & Square & Component & Component & Error \\
\hline $\mathrm{ENV}^{a}$ & 4 & 6865.0 & 170.6 & 170.6 & 121.4 \\
\hline ENTRY & 19 & 29.6 & 0.5 & 0.5 & 1.1 \\
\hline ENTRY*ENV & 76 & 11.5 & 3.5 & 3.5 & 1.0 \\
\hline $\mathrm{HABIT}^{b}$ & 1 & 81.7 & - & - & - \\
\hline $\mathrm{HABIT}^{*} \mathrm{ENV}^{a}$ & 4 & 33.9 & 1.5 & 1.5 & 1.2 \\
\hline HABIT*ENTRY & 19 & 17.3 & 2.5 & 2.5 & 1.1 \\
\hline $\mathrm{HABIT}^{*} \mathrm{ENTRY}^{*} \mathrm{ENV}^{c}$ & 76 & 4.6 & 4.6 & 4.6 & 0.7 \\
\hline
\end{tabular}

${ }^{a}$ Variance component estimated only when considered random

${ }^{b}$ Fixed effect-no variance component estimated

${ }^{c}$ In MOB approach, this effect is the error/residual term

Table 5: P-values for tests of effects from MOB approach

\begin{tabular}{|c|c|c|c|c|c|}
\hline \multirow[b]{3}{*}{ Source } & \multicolumn{3}{|c|}{ ENV Random } & \multicolumn{2}{|c|}{ ENV Fixed $^{a}$} \\
\hline & \multirow[b]{2}{*}{ GLM } & \multicolumn{2}{|c|}{ MIXED } & \multirow[b]{2}{*}{ GLM } & \multirow[b]{2}{*}{ MIXED } \\
\hline & & \multicolumn{2}{|l|}{ Likelihood } & & \\
\hline ENV & $<.01$ & $<.01$ & .16 & $<.01^{b}$ & $<.01$ \\
\hline ENTRY & .30 & .62 & .64 & & \\
\hline ENTRY*ENV & $<.01$ & $<.01$ & $<.01$ & & \\
\hline HABIT & .23 & \multicolumn{2}{|c|}{$.26^{c}$} & $.04^{b}$ & .04 \\
\hline $\mathrm{HABIT}^{*} \mathrm{ENV}^{a}$ & $<.01$ & $<.01$ & .22 & $<.01$ & $<.01$ \\
\hline HABIT*ENTRY & $<.01$ & $<.01$ & .02 & & \\
\hline
\end{tabular}

\footnotetext{
${ }^{a} \mathrm{P}$-values are presented only for effects for which test statistics change

${ }^{b}$ Requires that ENV*HABIT effect is zero

${ }^{c}$ PROC MIXED test for fixed effects does not depend on method chosen for testing random
} effects 
Table 6: REML variance component estimates for BE approach

\begin{tabular}{l|rrrrr}
\hline & \multicolumn{5}{|c}{ Environment } \\
\hline Source & ASH90 & HES91 & HES92 & OTT91 & OTT92 \\
\hline BLOCK & $<0$ & 1.0 & $<0$ & 10.7 & $4.8^{a}$ \\
ENTRY & $2.4^{a}$ & 2.8 & $2.5^{a}$ & 6.0 & $2.8^{a}$ \\
ENTRY*BLOCK $^{*}$ BABIT & $2.7^{a}$ & 4.5 & $0.8^{a}$ & 2.4 & $<0$ \\
HABIT $^{*}$ ENTRY & - & - & - & - & - \\
ERROR/RESIDUAL $^{*}$ & 8.5 & 7.3 & 3.4 & 1.8 & $4.0^{a}$ \\
& 6.4 & 4.2 & 4.4 & 5.0 & $11.3^{a}$
\end{tabular}

${ }^{a}$ Method of Moments estimate of this variance component differs slightly

${ }^{b}$ Fixed effect-no variance component estimated

Table 7: GLM p-values for tests of effects from BE approach

\begin{tabular}{l|rrrrr}
\hline & \multicolumn{5}{|c}{ Environment } \\
\hline Source & ASH90 & HES91 & HES92 & OTT91 & OTT92 \\
\hline BLOCK & .61 & .03 & .65 & $<.01$ & $<.01$ \\
ENTRY & .22 & .16 & .07 & $<.01$ & .14 \\
ENTRY $^{*}$ BLOCK & .02 & $<.01$ & .16 & .01 & .54 \\
HABIT $^{*}$ & .60 & $<.01$ & .51 & .01 & .59 \\
HABIT $^{*}$ ENTRY & $<.01$ & $<.01$ & $<.01$ & .02 & .03
\end{tabular}

Table 8: Likelihood Ratio p-values for tests of effects from BE approach

\begin{tabular}{|c|c|c|c|c|c|}
\hline & \multicolumn{5}{|c|}{ Environment } \\
\hline Source & ASH90 & HES91 & HES92 & OTT91 & OTT92 \\
\hline BLOCK & 1.00 & .09 & 1.00 & $<.01$ & $<.01$ \\
\hline ENTRY & .44 & .33 & .14 & $<.01$ & .25 \\
\hline ENTRY*BLOCK & .05 & $<.01$ & .35 & .04 & 1.00 \\
\hline $\mathrm{HABIT}^{a}$ & .60 & $<.01$ & .51 & .01 & .59 \\
\hline HABIT*ENTRY & $<.01$ & $<.01$ & $<.01$ & .05 & .03 \\
\hline
\end{tabular}

${ }^{a}$ Fixed effects are tested with an F-test 
Table 9: Wald p-values for tests of effects from BE approach

\begin{tabular}{l|rrrrr}
\hline & \multicolumn{5}{|c}{ Environment } \\
\hline Source & ASH90 & HES91 & HES92 & OTT91 & OTT92 \\
\hline BLOCK &.$^{a}$ & .45 &.${ }^{a}$ & .33 & .35 \\
ENTRY & .46 & .38 & .18 & .04 & .27 \\
ENTRY $^{*}$ BLOCK & .07 & $<.01$ & .36 & .06 &.$a$ \\
HABIT $^{b}$ & .60 & $<.01$ & .51 & .01 & .59 \\
HABIT $^{*}$ ENTRY & .01 & .01 & .04 & .12 & .12
\end{tabular}

\footnotetext{
${ }^{a}$ Test not performed since variance component $<0$

${ }^{b}$ Fixed effects are tested with an F-test; these are identical to the results from Table 9
}

Table 10: Means for the two HABIT groups for each environment

\begin{tabular}{|c|c|c|c|c|c|}
\hline & \multicolumn{5}{|c|}{ Environment } \\
\hline HABIT & ASH90 & HES91 & HES92 & OTT91 & OTT92 \\
\hline Indeterminate & 44.6 & 23.6 & 29.5 & 14.6 & 41.7 \\
\hline Determinate & 45.1 & 19.4 & 29.1 & 12.9 & 41.1 \\
\hline Standard Error ${ }^{a}$ & 0.7 & 0.7 & 0.5 & 0.4 & 0.6 \\
\hline
\end{tabular}

${ }^{a}$ Standard error is computed by hand using the appropriate denominator error term (HABIT*ENTRY) from PROC GLM 